\title{
Screening CT Colonography: How I Do It
}

Perry J. Pickhardt ${ }^{1}$

Keywords: colorectal cancer, colorectal polyps, CT colonography, screening, virtual colonoscopy

\section{DOI:10.2214/AJR.07.2136}

Received December 15, 2006; accepted after revision March 28, 2007.

P. J. Pickhardt's status as a consultant for Viatronix, Medicsight, and Fleet did not alter the content of this perspective.

'Department of Radiology, University of Wisconsin Medical School, E3/311 Clinical Science Center, 600 Highland Ave., Madison, WI 53792-3252. Address correspondence to P. J. Pickhardt (pj.pickhardt@hosp.wisc.edu).

\section{AJR 2007; 189:290-298}

0361-803X/07/1892-290

(c) American Roentgen Ray Society

OBJECTIVE. The purpose of this article is to detail an approach to CT colonographic screening that has evolved at one institution.

CONCLUSION. CT colonography is a rapidly advancing technology that has great potential for addressing a deadly but preventable disease - colorectal carcinoma. CT colonography is ideally suited for widespread screening of asymptomatic adults and has become an integral component of the screening efforts at my institution since local third-party coverage was initiated.

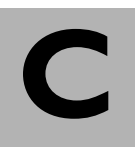

T colonography (CTC), also called virtual colonoscopy, is ideally suited for population screening of asymptomatic adults. Colorectal cancer, despite being largely preventable or at least curable if detected early, remains a major killer because of poor adherence to existing screening strategies. CTC screening is less invasive, less time-consuming, and less expensive than optical colonoscopy and can be at least as effective [1-3]. At my institution, the use of a previously validated CT screening method paved the way for local third-party reimbursement for screening of persons without symptoms, which was initiated in April 2004 [1, 2]. Since that time, more than 4,000 asymptomatic adults have undergone screening, and the initial results indicate that we have improved our earlier techniques [2]. Unfortunately, the current lack of third-party coverage elsewhere has stalled the logical transition of CTC from a research tool and limited diagnostic test to a full-fledged examination for widespread population screening. However, expanded coverage and use of CTC screening are on the horizon.

This review details the technique and basic interpretive approach for CTC screening at my institution. Other practical issues, such as program setup, patient referral, third-party reimbursement, extracolonic findings, cost-effectiveness, and common diagnostic pitfalls, are described elsewhere [3-7]. This work is expected to serve as a template for radiologists who are interested in but not yet fully engaged in CTC screening.

\section{CTC Technique}

The basic concept behind successful CTC is straightforward: When a properly prepared and distended colon is imaged with MDCT, clinically relevant polyps can be readily detected with dedicated CTC software [8]. If all facets of a CTC examination (preparation, distention, MDCT scanning, and interpretation) are adequately addressed, effective evaluation is relatively easy. Although each technical component is discussed separately, it is crucial to recognize the interdependence of these factors. Any weak link can render the overall product ineffective. For example, even the best CTC software system will fail if colonic preparation or distention is inadequate. Similarly, optimal preparation and distention cannot compensate for an inadequate CTC software system or an ineffective interpretive approach.

\section{Colonic Preparation}

Robust colonic preparation for CTC is critical for accurate polyp detection and involves both cleansing and tagging. My colleagues and I use a low-volume CTC preparation with three basic components: a laxative for catharsis, dilute barium for fecal tagging, and iodinated water-soluble contrast medium (diatrizoate) for fluid tagging (Table 1). This 1-day preparation has been greatly simplified from the one used in the Department of Defense screening trial and is generally well tolerated [9]. We have not encountered significant preparation-related complications. In addition to ingesting the agents prescribed for the preparation, the patient maintains a clear liquid diet the day before the examination. Be- 
TABLE I: Bowel Preparation for CT Colonography

\begin{tabular}{l|l}
\hline Time Range & \multicolumn{1}{|c}{ Oral Dose Instruction } \\
\hline $3-5 \mathrm{pm}$ & $45 \mathrm{~mL}$ of sodium phosphate \\
$6-8 \mathrm{pm}$ & $250 \mathrm{~mL}$ of $2 \%$ barium \\
$9-11 \mathrm{pm}$ & $60 \mathrm{~mL}$ of diatrizoate \\
\hline
\end{tabular}

Note-Preparation is undertaken in conjunction with a clear liquid diet the day before CT colonography. The steps are separated by approximately $3 \mathrm{~h}$. For the magnesium citrate alternative, the patient takes a $296-\mathrm{mL}$ bottle at both 3-5 pm and 6-8 pm. For the 4-L polyethylene glycol alternative, the patient begins the laxative at approximately noon to allow for the larger volume.

cause the preparation includes both over-thecounter laxatives and prescription oral contrast agents, we have our central pharmacy combine the constituents into a convenient kit, which is mailed to patients or dispensed at a number of local pharmacies [3].

The choice of laxative depends on the health status of the patient. Although the nurse coordinator was initially screening all patients to determine the most appropriate preparation [2], we have found it more efficient and logical to provide referring physicians with our guidelines and have them choose the best preparation for their patients. The laxative for our standard CTC bowel preparation is sodium phosphate, which is used in nearly $90 \%$ of cases and is well tolerated. Because of rare reported instances of acute phosphate nephropathy [10], we avoid the use of sodium phosphate by elderly patients with hypertension, particularly those taking angiotensin-converting enzyme inhibitors, and by patients with renal or cardiac insufficiency. We have found that a single $45-\mathrm{mL}$ dose of sodium phosphate is as effective as a double dose [9] and brings our preparation into U.S. Food and Drug Administration (FDA) compliance. With regard to a noncathartic CTC preparation, a number of disadvantages will likely prevent this approach from becoming a stand-alone frontline screening solution [11].

For nearly all patients who should avoid sodium phosphate, magnesium citrate is an acceptable substitute. Because we find magnesium citrate somewhat less effective than sodium phosphate, we use two 296-mL doses. For severely compromised patients who cannot tolerate even mild fluid or electrolyte shifts, we resort to polyethylene glycol (PEG), which is given as a $4-\mathrm{L}$ solution. Although the safety profile of polyethylene glycol is most favorable for patients with a tenuous condition, preparation with polyethylene glycol is associated with the poorest adherence because of the consistency and taste of the solution and the large volume that must be ingested. Fortunately, polyethylene glycol preparation is rarely necessary for screening CTC, accounting for less than $1 \%$ of cases at my institution.

Regardless of the laxative used, the dual oral contrast regimen remains constant, consisting of single doses of $2 \%$ weight/volume barium and diatrizoate. The complementary actions of these two contrast agents result in optimal preparation for CTC [12]. The rationale behind the specific order of the three components of the preparation is that the laxative provides catharsis for bulk removal of fecal material, the barium tags residual feces, and the diatrizoate serves the dual purpose of uniform fluid tagging and secondary catharsis [12]. In my opinion, the 30-40\% weight/volume barium preparations are too dense for CTC and may cause difficulty during same-day colonoscopy. In my experience, $2 \%$ barium has not caused problems in more than 1,500 colonoscopic studies performed immediately after CTC [13]. The ionic iodinated contrast agent not only opacifies luminal fluid and has an effect somewhat similar to that of cathartics but also helps emulsify residual solid feces. The last feature is critical because adherent feces can severely complicate primary $3 \mathrm{D}$ polyp detection. The limited experience with nonionic iodinated contrast material (e.g., iohexol) at my institution suggests that the greater palatability of the nonionic agent does not override the problem of a large amount of adherent residual fecal material.

Oral contrast tagging for CTC increases specificity by tagging residual fecal material (barium effect) and decreasing the amount of adherent feces (diatrizoate effect). It increases sensitivity by allowing polyp detection in opacified fluid (diatrizoate effect). A potential pitfall that has become an interpretive asset is the tendency for true soft-tissue polyps to retain a thin surface coating of contrast material [14], which serves as a beacon for detection (Fig. 1). Given the improved performance characteristics associated with use of oral contrast material, my colleagues and I strongly endorse use of these agents for CTC screening. With regard to computeraided detection, most algorithms were not developed with contrast tagging in mind. Going foward, this aspect of the imaging procedure must be addressed $[15,16]$.

\section{Colonic Distention}

Adequate distention of the colon, like proper bowel cleansing, is critical to technical success. Adequate colonic distention is generally distinct from maximal distention, because patient comfort and safety must be taken into account if CTC is to be widely embraced. With the protocol used at my institution, inadequate segmental distention occurs in less than $1 \%$ of cases. In general, gaseous distention for CTC can be achieved with either room air or $\mathrm{CO}_{2}$, and the rate and degree of insufflation can be automated or manually controlled by either the patient or the medical staff (technologist or physician). Rigid largecaliber retention balloon catheters designed for barium enemas are seldom necessary. For most patients undergoing screening, smaller and more flexible rectal catheters are preferred for patient comfort and safety. Spasmolytic agents are not used because the disadvantages (e.g., added invasiveness, risk of side effects, increased duration of the examination, and increased costs) outweigh the uncertain benefit [17].

My colleagues and I have found that automated $\mathrm{CO}_{2}$ delivery is the best distention technique. Nearly all reported perforations during CTC have involved the use of staff-controlled manual insufflation of room air. The risk of perforation with automated or patient-controlled distention methods approaches zero among asymptomatic adults [18]. With regard to both study quality (i.e., degree of distention) and postprocedural discomfort, we have found that dynamic $\mathrm{CO}_{2}$ delivery is superior to manual administration of room air [17]. Continuous lowpressure automated $\mathrm{CO}_{2}$ delivery appears to reduce colonic spasm, particularly in segments with advanced diverticular disease. Rapid resorption of $\mathrm{CO}_{2}$ through the colonic wall accounts for improved comfort after the procedure. The CT technologists at my institution unanimously prefer the automated $\mathrm{CO}_{2}$ technique over manual methods [17]. They cite a more clearcut point to begin scanning, a diminished need for coaching patients, and decreased operator dependence, which leads to more consistent results from technologist to technologist.

To maintain efficiency, the CT technologists at my institution are trained to independently perform the entire CTC examination, including placement of the rectal catheter and assessment of adequacy of distention. The radiologist is consulted for difficult or unusual situations, which allows more time for interpretation. Before beginning an examination, the technologist interviews the patient to de- 


\section{Pickhardt}
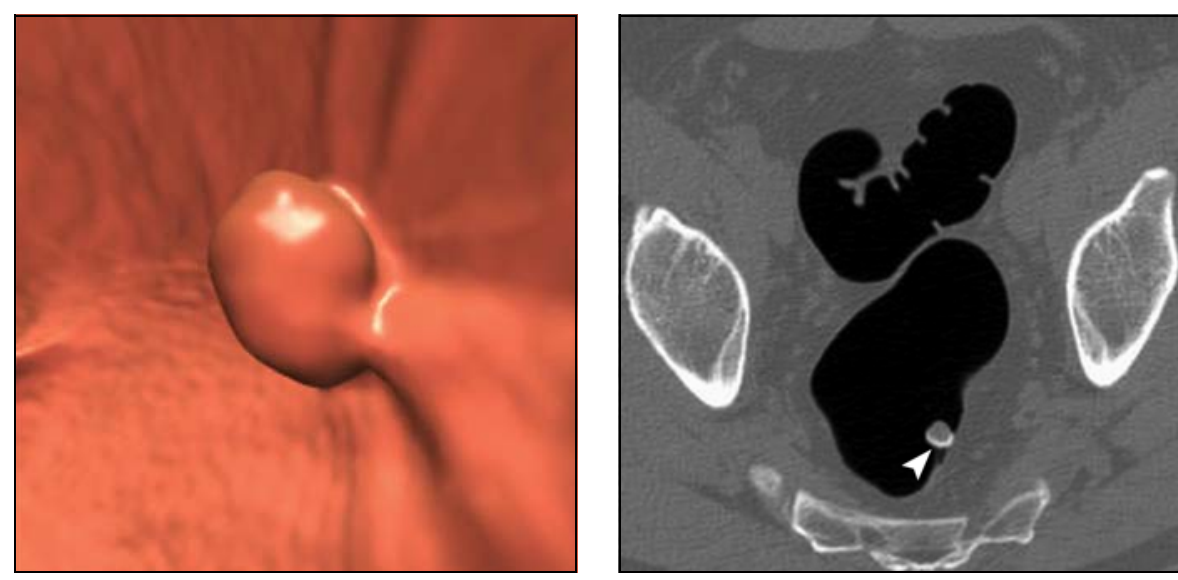

A
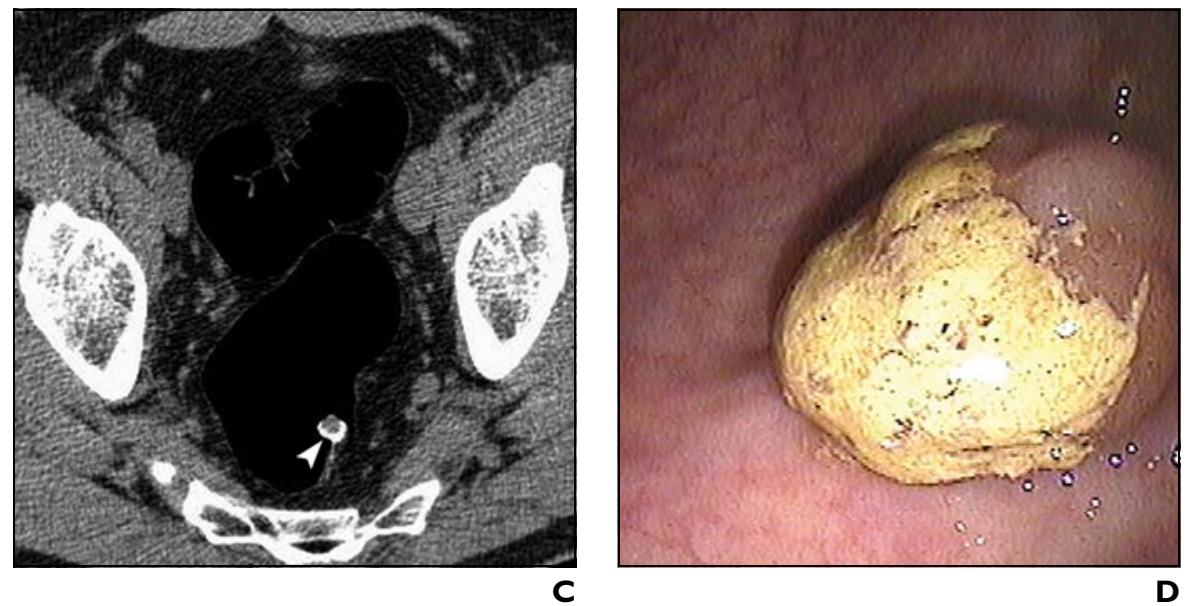

Fig. 1-Contrast coating of polyp surface on screening CT colonography in 56-year-old man.

A, Three-dimensional endoluminal colonographic image shows 1.5 -cm sessile polyp within rectum.

B and C, Two-dimensional transverse CT colonographic images with polyp (B) and soft-tissue (C) window settings show lesion of uniform soft-tissue density with rim of adherent contrast material coating posterior surface (arrowhead), which should not be confused with fecal tagging. Contrast material appears to enlarge or "bloom" in C, decreasing conspicuity of polyp. Polyp windows are important for appreciating homogeneous nature of internal soft-tissue component of these lesions.

D, Digital photograph from optical colonoscopy shows tenacious mucus clinging to portion of polyp surface, which may correspond to dense coating at CT colonography. Polyp proved to be tubulovillous adenoma. Lesions with villous component tend to have this coating effect.

termine the fidelity of preparation, and the patient is encouraged to use the restroom. After rectal catheter placement, the patient remains in the left lateral decubitus position for delivery of the initial $1-1.5 \mathrm{~L}$ of $\mathrm{CO}_{2}$ with the automated device $\left(\mathrm{PROTOCO}_{2} \mathrm{~L}, \mathrm{E}-\mathrm{Z}-\mathrm{EM}\right)$. To reduce the transient discomfort related to initial rectal distention, the equilibrium pressure is initially set to $20 \mathrm{~mm} \mathrm{Hg}$. The patient is then placed in the right lateral decubitus position until a total of approximately $2.5 \mathrm{~L}$ of $\mathrm{CO}_{2}$ has the been delivered. The patient assumes the supine position until steady-state equilibrium has been reached, and then scanning is begun. The positional change helps to prevent inadequate distention related to the presence of a fluid channel or bowel kink.

The volume of $\mathrm{CO}_{2}$ dispensed can vary widely not only because of differences in actual colonic volume but also because of varying degrees of reflux through the ileocecal valve, loss around the catheter, and continuous colonic resorption. Therefore, the total volume reading for $\mathrm{CO}_{2}$ delivery has relatively little meaning and can range from $3 \mathrm{~L}$ to more than $10 \mathrm{~L}$. It is imperative to acquire the MDCT images during active replacement of $\mathrm{CO}_{2}$ at equilibrium. The supine and prone scans are obtained at end expiration to raise the diaphragm, allowing more room for the splenic flexure and transverse colon.
Although the scout image gives a general indication of distention adequacy, it is unreliable for assessment of the left colon [19] (Fig. 2). The CT technologists at my institution are trained to recognize inadequate distention, particularly of the sigmoid colon, by online review of $2 \mathrm{D}$ transverse images with a wide window setting. This step ensures adequate distention of the left colon and avoids the need for calling the patient back. If focal collapse persists at the same point on both supine and prone scans, a series of right lateral decubitus images are obtained (Fig. 2), which often yields sufficient information for diagnosis. In less than $1 \%$ of patients, segmental evaluation is inadequate despite all efforts. On rare occasion, to complete the screening evaluation we have offered patients same-day flexible sigmoidoscopy without sedation.

\section{MDCT Protocol}

Compared with temporally demanding protocols such as CT angiography, CTC is a forgiving examination with regard to scanner requirements. Screening CTC is performed without contrast enhancement, the gas-filled large intestine is a relatively static structure, and the minimum target size is a 6-mm lesion. Consequently, submillimeter collimation is not needed to perform state-of-the-art CTC, and 8 - or 16-MDCT is more than adequate. The increase in the dose and number of images required for submillimeter collimation to balance image noise outweighs any theoretic benefit for polyp detection. Most CTC studies for the Department of Defense screening trial were performed on 4-MDCT scanners with $4 \times 2.5 \mathrm{~mm}$ detector configuration and a 1-mm reconstruction interval [1]. $\mathrm{Al}$ though $1.25-\mathrm{mm}$ collimation is preferred, this technique is adequate for CTC.

The CTC scanning protocol at my institution is relatively straightforward. Regardless of continuing advances in CTC technique and interpretation, the established practice of obtaining both supine and prone scans remains firmly entrenched because of the invaluable complementary data obtained. Typical 8 - and 16-MDCT scanning factors include $1.25-\mathrm{mm}$ collimation, 1.0 - to $1.25-\mathrm{mm}$ reconstruction interval, and $120 \mathrm{kVp}$. Given the nature of the soft tissue-air interface for polyp detection during CTC, the radiation dose can be markedly less than the usual diagnostic level. To optimize the delivered dose, my colleagues and I use a tube current modulation system (Smart mA, GE Healthcare) that modulates current in both the $x y$-plane and the $z$-plane. 


\section{Screening CT Colonography}

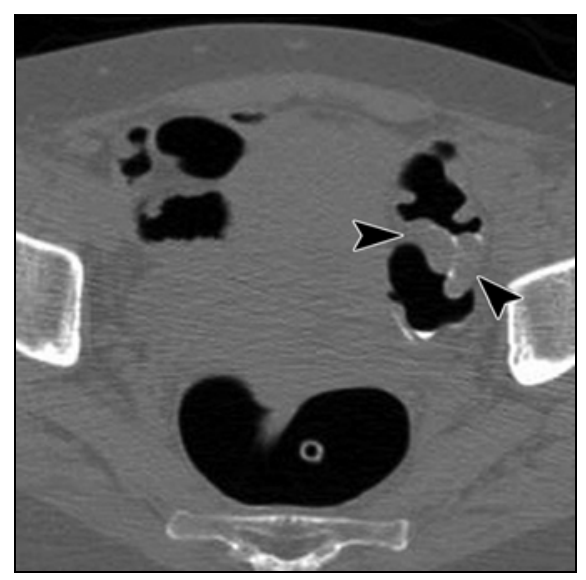

A

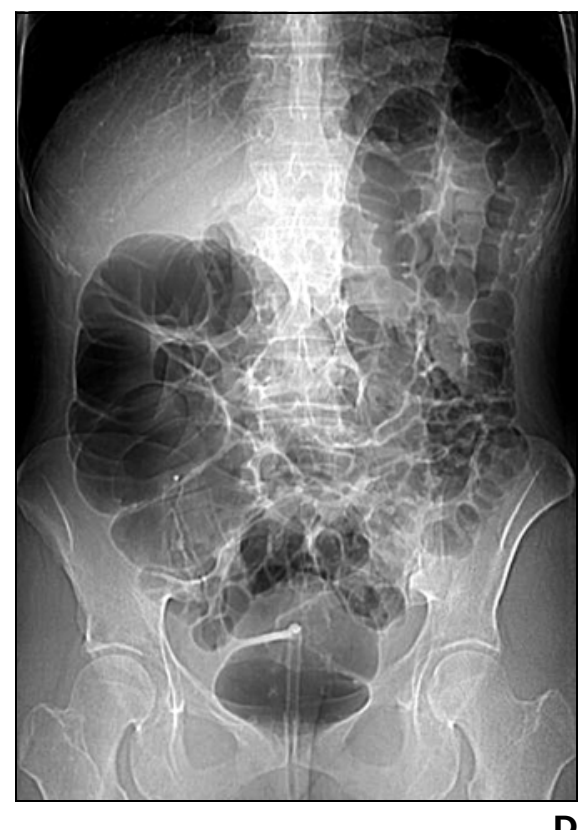

We set the noise index at 50 and the tube current range at 30-300 $\mathrm{mA}$, which has yielded considerable dose reduction and examinations of consistent quality for diagnosis. For MDCT scanners not equipped with a tube current modulation system, technique with a tube current-time product in the range of 50-75 mAs suffices, except for some morbidly obese patients. Although further dose reduction is a desirable goal for evaluation of adults who do not have symptoms, the small theoretic risk of low-dose radiation exposure is clearly outweighed by the benefit of colorectal cancer screening [20,21].

With the scanning parameters used at my institution, each series has fewer than 500 images, and fewer than 1,000 images typi-
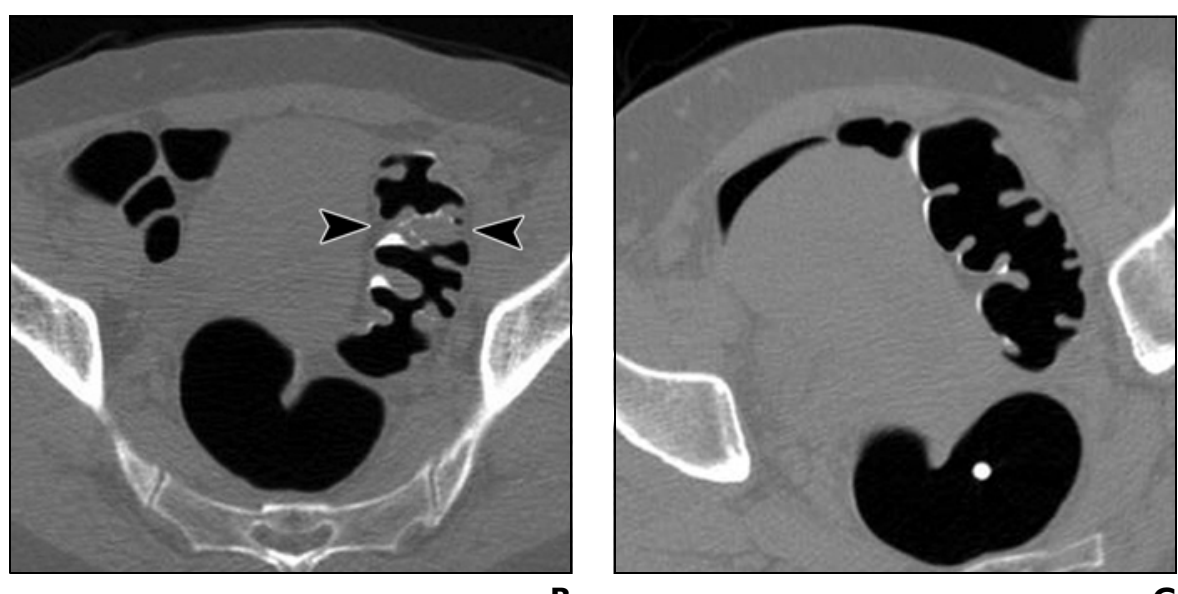

Fig. 2-Addition of decubitus view because of inadequate sigmoid distention with supine and prone positioning in 53-year-old woman.

A and B, Supine (A) and prone (B) 2D transverse CT colonographic images show focal segmental collapse (arrowheads) and fold thickening involving sigmoid colon. Distention was adequate throughout remainder of large intestine (not shown). In immediate review of 2D images, CT technologist recognized collapse at same position on both supine and prone images and obtained additional right lateral decubitus image.

C, Two-dimensional transverse CT colonographic image obtained with patient in right lateral decubitus position shows adequate distention of segment in question, allowing diagnostic examination and avoiding need for flexible sigmoidoscopy.

D, Prone scout radiograph does not show area of focal collapse, in part because presence of overlapping loops makes assessment difficult. Finding on scout image can lead to both overestimation and underestimation of distention and necessitates online review of $2 \mathrm{D}$ transverse images by $\mathrm{CT}$ technologist or radiologist for confident assessment of left colonic distention.

cally are obtained for the total study. These source images are sent to the CTC workstation for advanced modeling and interpretation and to a PACS for storage. To facilitate extracolonic evaluation, in addition to the thin-section source data, a series of contiguous 5-mm images is reconstructed from the supine image data set [5].

\section{CTC Interpretation}

\section{D and 3D Polyp Detection}

Both 2D and 3D visualization modes should be used for polyp detection, although the latter is much easier and more effective for low-prevalence screening. Interpretive redundancy on different views ensures accurate polyp detection by adding complementary evaluation. Primary 2D evaluation alone, 3D being reserved for problem solving, is an inadequate interpretive approach to low-prevalence CTC screening, primarily because it does not incorporate the benefit of primary 3D detection. The biphasic interpretive approach used at my institution emphasizes 3D detection but also retains the added value of $2 \mathrm{D}$ detection. The underlying rationale is simple: The more sensitive but less specific display (3D endoluminal) is best for initial polyp detection, and the more specific but less sensitive display (2D) is needed for confirmation of suspected lesions and, in some cases, auxiliary polyp detection. Although 3D polyp detection is usually comprehensive, $2 \mathrm{D}$ evaluation can be particularly useful in the presence 


\section{Pickhardt}
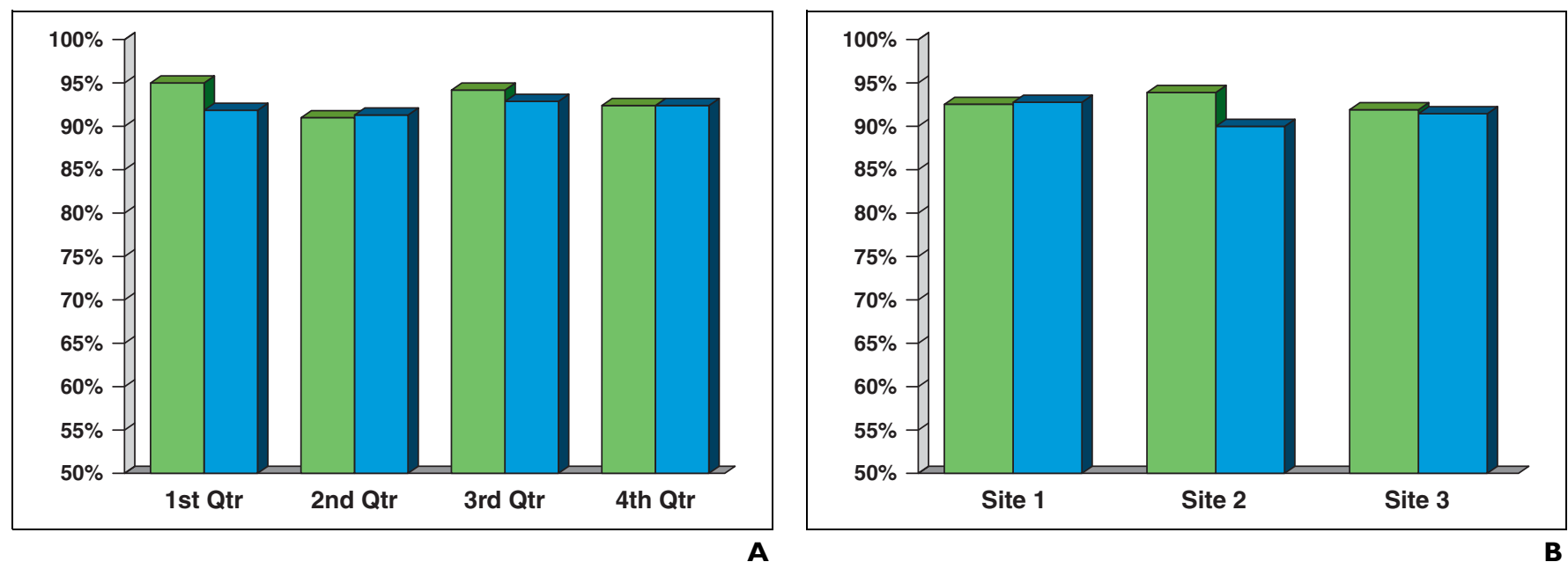

Fig. 3-Performance of 3D CT colonography in detection of adenomas $8 \mathrm{~mm}$ or larger in Department of Defense CT colonography screening trial [1].

A and B, Bar graphs show sensitivity (green) and specificity (blue) at 8-mm size threshold according to temporal quarter (Qtr) of trial (A) and study site (B). Uniform performance characteristics range from $91 \%$ to $95 \%$ in all cases. That most radiologists in study were not based at academic medical centers and had relatively little experience (25-50 of fewer CT colonography cases) suggests that learning curve with primary 3D polyp detection is much simpler than that for primary 2D approach. Site 1 = National Naval Medical Center, site 2 = Naval Medical Center San Diego, site 3 = Walter Reed Army Medical Center.

of abundant adherent feces or segments with partial or total luminal collapse. For 2D polyp detection, we prefer a window width of 2,000 $\mathrm{H}$ centered at $0 \mathrm{H}$.

Among the large CTC trials involving cohorts with a low prevalence of disease, protocols restricted to a primary $2 \mathrm{D}$ approach fared poorly [22-24], whereas those that relied primarily on 3D polyp detection performed well $[1,25]$. The use of oral contrast tagging in the 3D trials probably imparted some benefit. Not surprisingly, retrospective primary 2D evaluation of cases from our screening trial has shown a significant reduction in sensitivity for polyp detection compared with prospective 3D interpretation (unpublished data). Furthermore, review of the false-negative CTC results from the 2D trial conducted by Rockey et al. [24] showed that most missed lesions were retrospectively identified with CTC. My colleagues and I consider it highly probable that most of the lesions missed with 2D imaging in the study by Doshi et al. [26] would have been detected prospectively with adequate $3 \mathrm{D}$ evaluation.

The 3D endoluminal approach works well because polyp conspicuity among the folds is greatly enhanced compared with 2D visualization. The result is a much easier learning curve [27, 28] (Fig. 3). At primary 2D evaluation, the ubiquitous haustral folds have a somewhat polypoid appearance, which adds to the challenge of the search for polyps. It seems impractical to struggle to find, or miss, a polyp in $2 \mathrm{D}$ when most polyps are obvious on the $3 \mathrm{D}$ endoluminal view. Although one solution to poor $2 \mathrm{D}$ sensitivity is to incorporate computer-aided detection, this approach has yet to match the performance of primary $3 \mathrm{D}$ evaluation $[1,20]$. Reviewer fatigue and eyestrain resulting from a $2 \mathrm{D}$ polyp search are important issues to consider with high-volume CTC screening. My colleagues and I have found that radiologists generally begin as 2D CTC reviewers but gravitate toward 3D polyp detection as their experience and the volume of polyps detected grow.

Although not yet validated for screening, emerging 3D displays beyond the standard endoluminal projection deserve mention. The virtual dissection, or filet, view has received the most attention [29, 30]. The promise of this display lies in the potential for reducing 3D interpretation time because bidirectional navigation is not needed. The compromise with these novel 3D projections is spatial distortion whereby some polyps become unrecognizable. The effect of this technique on accuracy remains uncertain. My colleagues and I are investigating a variety of primary $3 \mathrm{D}$ solutions in a multivendor comparison trial. Optimizing the standard endoluminal field of view to balance mucosal coverage and visual distortion is another potential improvement that is under investigation. This approach appears to allow unidirectional 3D endoluminal fly-through without sacrificing polyp detection (unpublished data).

\section{Interpretive Approach}

The CTC interpretation system (V3D, Viatronix) used at my institution is validated for colorectal screening and is FDA-approved for this purpose [1,3]. Software improvements with this system have resulted in faster and more accurate CTC interpretation [2, 31]. Although the 3D capabilities of most other CTC systems were not originally devised for effective, time-efficient primary $3 \mathrm{D}$ evaluation [32], several systems are rapidly advancing. It should be only a matter of time before validation and FDA approval are achieved for other CTC systems.

The supine and prone source images are sent to the CTC software system for interpretation, which segments out the gas-filled bowel and generates an automated centerline. For most screening cases, I begin with 3D endoluminal evaluation because almost all significant polyps are readily detectable with this display. Primary 3D endoluminal review entails bidirectional fly-through of the supine and prone models, which is largely hands-free along the automated centerline and interspersed with manual navigation as needed for interrogating potential abnormalities. My colleagues and I are investigating whether a field of view increased beyond $90^{\circ}$ alleviates the need for four complete fly-through views. Flight time from rectum to cecum and vice versa currently is approximately 1 minute in a typical case. Although the CTC system used at my institution allows electronic cleansing, 


\section{Screening CT Colonography}

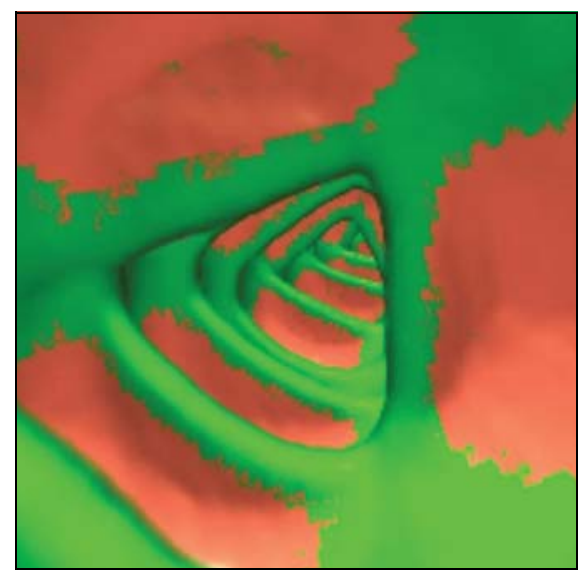

Fig. 4-Mucosal coverage on 3D endoluminal CT colonography in 59-year-old woman. Still image from $3 \mathrm{D}$ endoluminal fly-through shows visualized surface (green) after unidirectional navigation along automated centerline in opposite direction. Typically, more than $20 \%$ of endoluminal surface is not seen during unidirectional navigation with $90^{\circ}$ field of view, necessitating bidirectional navigation. Previously unseen areas are relative blind spots at optical colonoscopy. Nonstandard 3D displays, such as virtual dissection view, avoid bidirectional evaluation and may become standard practice. Increasing field of view may also eliminate need for bidirectional endoluminal navigation.

or digital subtraction, of opacified residual luminal fluid [12], my colleagues and I keep this function disabled because we believe that the theoretic benefits are outweighed by the artifacts introduced [9, 31].

The CTC system continuously tracks the visualized endoluminal surface (Fig. 4). A missed-region tool allows the reviewer to click through the mucosal patches not seen during routine bidirectional centerline navigation, which generally increases coverage to $98-99 \%$, yet consumes only 10-30 seconds of interpretation time [33]. The ability to track 3D endoluminal coverage not only increases reviewer confidence but also avoids the need to start over if interpretation is interrupted. Interrogation of polypoid lesions detected on $3 \mathrm{D}$ views can be accomplished with the usual 2D multiplanar reconstruction correlation or with 3D translucency rendering (Fig. 5), which allows rapid evaluation of internal density [34]. When used properly, the translucency-rendering tool can decrease interpretation time by reducing the need for 2D confirmation of false-positive findings, particularly tagged feces. Some 3D endoluminal findings, however, require 2D correlation. In most cases, entire screening CTC studies can be interpreted with combined 3D and $2 \mathrm{D}$ evaluation in 10 minutes or less [31].
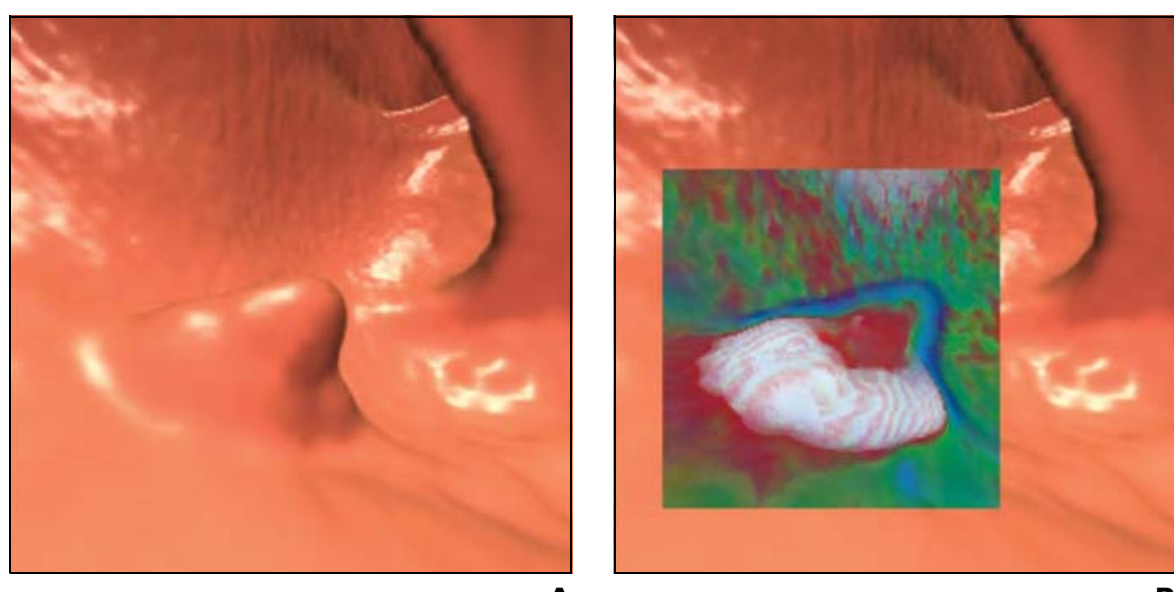

A

$\mathbf{B}$
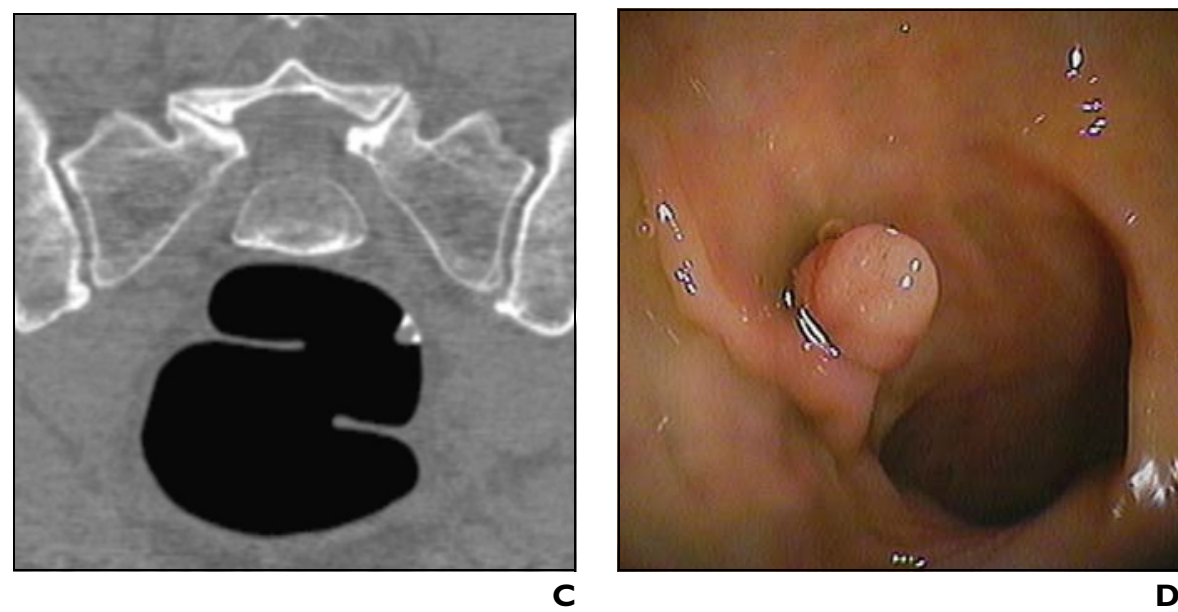

Fig. 5-Three-dimensional translucency rendering for assessment of internal density characteristics in 58-yearold woman.

A, Three-dimensional endoluminal CT colonographic image shows 7-mm polypoid lesion in rectum.

B, Same 3D image as A with translucency rendering shows lesion composed of soft tissue (red) surrounded by thick collar of adherent contrast material at base (white).

C, Two-dimensional coronal CT colonographic image confirms presence of rectal soft-tissue polyp with highattenuation contrast material at base.

D, Digital photograph from same-day optical colonoscopy shows polyp depicted in A-C. Lesion proved to be tubular adenoma. Collar of adherent contrast material probably was washed away before photograph was obtained.

When a nondiminutive lesion is detected and confirmed to be composed of soft tissue, a bookmark is placed. Most true polyps can be verified on the alternate supine or prone view, resulting in increased diagnostic confidence. Linear polyp measurement is not as straightforward as it may seem, because the 2D measurement, which should always be optimized among the multiplanar reconstruction views, tends be an underestimate of polyp size, and the $3 \mathrm{D}$ view sometimes produces an overestimate of size [35]. Therefore, my colleagues and I have found it useful to take both measurements into consideration before arriving at a final value. In the future, polyp volume assessment may play an important role in surveillance of unresected lesions [36]. In addition to polyp size, segmental location, morphologic type (pedunculated, sessile, or flat), and diagnostic confidence score are recorded for each polyp. The diagnostic confidence score can provide useful information for program quality assurance [37].

A screening CTC study is considered to have a positive result when a lesion $6 \mathrm{~mm}$ or larger is detected, although the polyps of greatest clinical significance are $10 \mathrm{~mm}$ or larger. My colleagues and I offer same-day optical colonoscopy to patients who have positive results on CTC, avoiding the need for additional preparation $[2,8]$. However, because the procedural risks associated with colono- 


\section{Pickhardt}

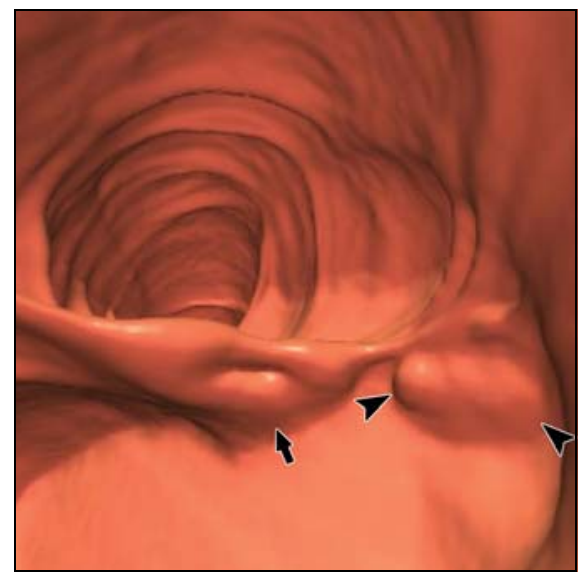

A

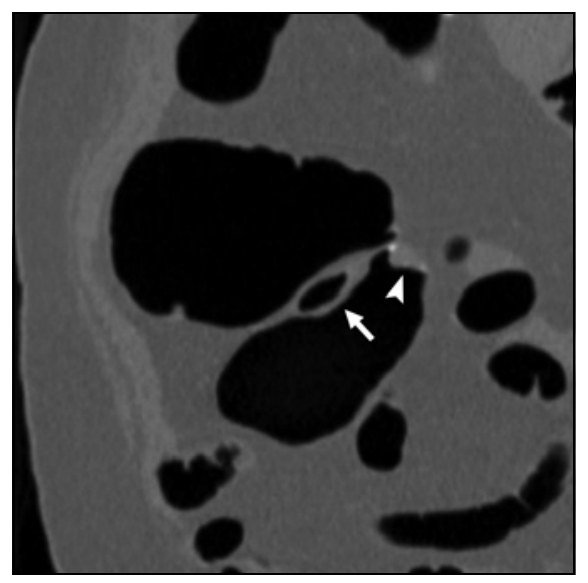

B

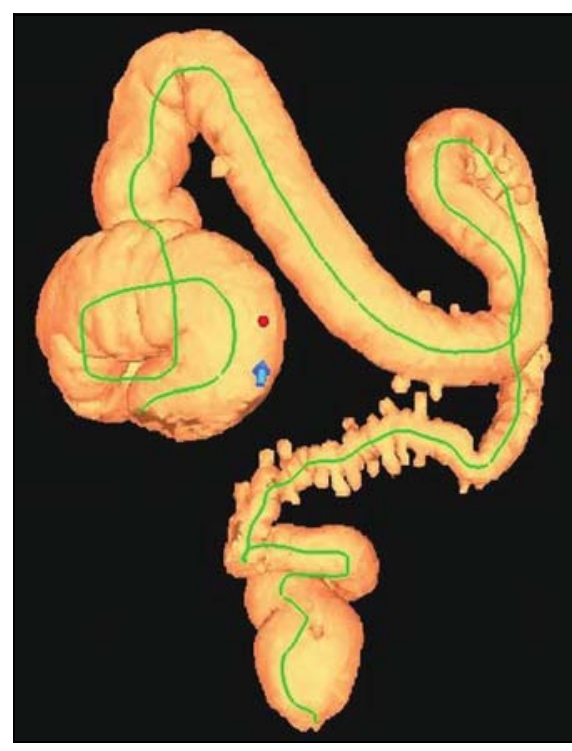

C

Fig. 6-Difficult polyp location for detection and polypectomy with optical colonoscopy in 68-year-old woman.

A, Three-dimensional endoluminal CT colonographic image from perspective of cecal tip shows relatively subtle 1.5-cm sessile polyp (arrowheads) located behind fold and adjacent to ileocecal valve (arrow).

B, Two-dimensional coronal CT colonographic image confirms presence of soft-tissue lesion (arrowhead) next to ileocecal valve (arrow).

C, Three-dimensional colonic map shows anatomic location of cecal polyp (red dot), extensive sigmoid diverticulosis, and automated centerline (green). Blue arrow indicates 3D vantage point shown in A. Polyp was found and removed at optical colonoscopy and proved to be tubulovillous adenoma with high-grade dysplasia. Because of difficult location of polyp, gastroenterologist noted that he would have missed this lesion without detailed knowledge of its existence obtained with CT colonography.

scopic polypectomy probably outweigh the malignant potential of a small polyp (6-9 $\mathrm{mm}$ ), we also offer the option of noninvasive CTC surveillance [2]. A consensus proposal [38] suggests a 3-year follow-up interval for CTC surveillance of one or two small polyps, and this recommendation is supported by natural history data [39, 40]. For colonoscopic referral, we provide the gastroenterologist with digital images showing the polyp location on the 3D map, 3D endoluminal projections, and 2D multiplanar reconstructions (Fig. 6). Special note is made of polyps located on the backside of folds, which are more easily missed at colonoscopy [41].

Nearly $90 \%$ of screening CTC studies at my institution have negative results [2], and we recommend routine screening in 5 years. For a number of reasons, the presence of diminutive lesions should not be mentioned. First, tiny polyps are not clinically relevant, yet mentioning them can cause undue anxiety in patients and referring physicians $[1,38,40$, 42]. Most diminutive "lesions" detected at CTC cannot be found at subsequent colonoscopy, representing either false-positive CTC findings or false-negative colonoscopic findings. To avoid any confusion or false pretenses, we include the following disclaimer in all CTC reports: "Note: CT colonography is not intended for the detection of diminutives polyps $(\leq 5 \mathrm{~mm})$, the presence or absence of which will not change the clinical management of the patient."

\section{Diagnostic Pitfalls}

CTC interpretation has a number of potential pitfalls (Appendix 1). Some pitfalls, such as prominent and complex folds, diverticular fold thickening, and shifting of pedunculated polyps, present more of a problem at 2D evaluation. Other pitfalls, such as annular masses, submucosal or extrinsic lesions, and impacted diverticula, are more an issue at $3 \mathrm{D}$ evaluation. With a biphasic interpretive approach, most pitfalls are easily recognized because of the complementary nature of the 2D and 3D displays.

Diverticular disease can frequently present a challenge in terms of achieving adequate distention and 2D polyp detection, but overall accuracy need not suffer with careful technique and the addition of primary $3 \mathrm{D}$ evaluation [43]. Likewise, although flat lesions are more challenging to detect initially, the sensitivity of CTC still appears adequate [44]. In addition, histologically advanced flat lesions appear to be rare in the screening population in the United States and do not represent a sig- nificant limitation of CTC screening [44, 45]. Carpet lesions are a subset of flat lesions that, despite their large linear dimensions, can be relatively subtle on CTC. My colleagues and I have found that classic large carpet lesions are rare but often prove to be villous adenomas, whereas flat lesions smaller than $3 \mathrm{~cm}$ tend to be nonneoplastic hyperplastic lesions.

An interesting observation is that most polyps of significant size missed with our CTC approach turn out to be nonadenomatous (predominately hyperplastic) even though such lesions are considerably outnumbered by adenomas at this size [46]. An isolated thickened fold can present a diagnostic challenge on CTC but rarely if ever represents a significant pathologic condition if it is smooth and uniform at 3D evaluation. Other pitfalls that we have encountered on a number of occasions relate to inverted appendiceal stumps, submucosal vascular blebs, the ileocecal valve, and the presence of the rectal catheter [34, 47-49].

\section{Conclusion}

With continued improvements in bowel preparation, colonic distention, and CTC interpretation, the results of CTC screening at my institution have exceeded initial expectations. 


\section{Screening CT Colonography}

The significant reduction in false-positive results has led to a $90 \%$ positive predictive value and has decreased the CTC test-positive rate to about $12 \%$ [2]. The increase in specificity has not been at the expense of decreased sensitivity. A direct comparison of CTC and optical colonoscopy programs showed similar detection rates for advanced neoplasia (Kim DH et al., presented at the 2006 meeting of the European Society of Gastrointestinal and Abdominal Radiology/Society of Gastrointestinal Radiologists). That the number of invasive procedures and polypectomies needed to achieve the same goal is many times less with the CTC approach shows that this examination is an effective and efficient filter for primary screening. Once national insurance coverage for CTC screening is in place, this procedure is poised to become a major practice component of our specialty. If the procedural details are properly addressed, CTC screening may have a profound effect on screening for and prevention of colorectal cancer.

\section{References}

1. Pickhardt PJ, Choi JR, Hwang I, et al. CT virtual colonoscopy to screen for colorectal neoplasia in asymptomatic adults. N Engl J Med 2003; 349:2191-2200

2. Pickhardt PJ, Taylor AJ, Kim DH, Reichelderfer M, Gopal DV, Pfau PR. Screening for colorectal neoplasia with CT colonography: initial experience from the first year of coverage by third-party payers. Radiology 2006; 241:417-425

3. Pickhardt PJ, Taylor AJ, Johnson GL, et al. Building a CT colonography program: necessary ingredients for reimbursement and clinical success. Radiology 2005; 235:17-20

4. Pickhardt PJ. CT colonography (virtual colonoscopy): a practical approach for population screening. Radiol Clin North Am 2007; 45:361-375

5. Pickhardt PJ, Taylor AJ. Extracolonic findings identified in asymptomatic adults at screening CT colonography. AJR 2006; 186:718-728

6. Hanson M, Pickhardt PJ, Kim DH, Taylor AJ, Vanness D. Unsuspected extracolonic findings at screening CT colonography: frequency of additional work-up and relevant pathology. (abstr) RSNA 2006. Oak Brook, IL: Radiological Society of North America, 2006. Available at: http:// rsna2006.rsna.org/rsna2006/V2006/conference/ event_display.cfm?em_id=4427572. Accessed April 30, 2007

7. Pickhardt PJ, Hassan C, Laghi A, Zullo A, Kim DH, Morini S. Cost-effectiveness of colorectal cancer screening with CT colonography: the impact of not reporting diminutive lesions. Cancer 2007;
2213-2221

8. Pickhardt PJ. Virtual colonoscopy for primary screening: the future is now. Minerva Chir 2005; 60:139-150

9. Kim DH, Pickhardt PJ, Hinshaw JL, Taylor AJ, Mukherjee R, Pfau PR. Prospective blinded trial comparing 45-ml and 90-ml doses of oral sodium phosphate for bowel preparation prior to CT colonography. J Comput Assist Tomogr 2007; 31:53-58

10. Markowitz GS, Stokes MB, Radhakrishnan J, D'Agati VD. Acute phosphate nephropathy following oral sodium phosphate bowel purgative: an underrecognized cause of chronic renal failure. J Am Soc Nephrol 2005; 16:3389-3396

11. Pickhardt PJ. CT colonography without catharsis: the ultimate study or useful additional option? Gastroenterology 2005; 128:521-522

12. Pickhardt PJ, Choi JR. Electronic cleansing and stool tagging in CT colonography: advantages and pitfalls encountered with primary three-dimensional evaluation. AJR 2003; 181:799-805

13. Pickhardt PJ. Virtual colonoscopy to screen for colorectal cancer. (reply) N Engl J Med 2004; 350:1148-1150

14. O'Connor SD, Summers RM, Yao J, Choi JR, Pickhardt PJ. Oral contrast adherence to polyps on CT colonography. J Comput Assist Tomogr 2006; 30:51-57

15. Summers RM, Franaszek M, Miller MT, Pickhardt PJ, Choi JR, Schindler WR. Computer-aided detection of polyps in oral contrast-enhanced CT colonography. AJR 2005; 184:105-108

16. Summers RM, Yao J, Pickhardt PJ, et al. Computed tomographic virtual colonoscopy computer-aided polyp detection in a screening population. Gastroenterology 2005; 129:1832-1844

17. Shinners TJ, Pickhardt PJ, Taylor AJ, Jones DA, Olsen $\mathrm{CH}$. Patient-controlled room air insufflation versus automated carbon dioxide delivery for CT colonography. AJR 2006; 186:1491-1496

18. Pickhardt PJ. The incidence of colonic perforation at CT colonography: review of the existing data and the implications for screening of asymptomatic adults. Radiology 2006; 239:313-316

19. Choi M, Taylor AJ, VonBerge JL, Bartels CM, Pickhardt PJ. Can the CT scout reliability assess for adequate colonic distention at CT colonography? (abstr) AJR 2005; 184 [American Roentgen Ray Society 105th Annual Meeting Abstract Book suppl]:21

20. Brenner DJ, Georgsson MA. Mass screening with CT colonography: should radiation exposure be of concern? Gastroenterology 2005; 129:328-337

21. [No authors listed]. Radiation risk in perspective: position statement of the Health Physics Society. Health Physics Society Website. Available at: http://hps.org/documents/risk_ps010-1.pdf. Ac- cessed May 16, 2007

22. Johnson CD, Harmsen WS, Wilson LA, et al. Prospective blinded evaluation of computed tomographic colonography for screen detection of colorectal polyps. Gastroenterology 2003; 125:311-319

23. Cotton PB, Durkalski VL, Pineau BC, et al. Computed tomographic colonography (virtual colonoscopy): a multicenter comparison with standard colonoscopy for detection of colorectal neoplasia. JAMA 2004; 291:1713-1719

24. Rockey DC, Paulsen EK, Niedzwiecki D, et al. Analysis of air contrast barium enema, computed tomographic colonography, and colonoscopy: prospective comparison. Lancet 2005; 365:305-311

25. Cash BD, Kim C, Cullen P, et al. Accuracy of computed tomographic colonography for colorectal cancer screening in asymptomatic individuals. (abstr) In: Digestive Disease Week 2006 annual meeting program. Bethesda, MD: DDW, 2006:473

26. Doshi T, Rusinak DJ, Halvorsen RA, Rockey DC, Dachman AH. Retrospective analysis of sources of error in a large CTC clinical trial. (abstr 098) AJR 2006; 186:A25-A27

27. Lee AD, Pickhardt PJ. Polyp visualization at CT colonography: comparison of 2D axial and 3D endoluminal displays. (abstr) RSNA 2004. Oak Brook, IL: Radiological Society of North America, 2004. Available at: http://rsna2004.rsna.org/rsna2004/ V2004/conference/event_display.cfm?em_id= 4409262. Accessed April 30, 2007

28. Pickhardt PJ, Choi JR, Nugent PA. Adenomatous polyps associated with colorectal folds at CT colonography: frequency and sensitivity for detection using a primary 3D approach. (abstr) RSNA 2004. Oak Brook, IL: Radiological Society of North America, 2004. Available at: http://rsna2004. rsna.org/rsna2004/V2004/conference/event_display. cfm?em_id=4405720. Accessed April 30, 2007

29. Juchems MS, Fleiter TR, Pauls S, Schmidt SA, Brambs H, Aschoff AJ. CT colonography: comparison of a colon dissection display versus 3D endoluminal view for the detection of polyps. Eur Radiol 2006; 16:68-72

30. Silva AC, Wellnitz CV, Hara AK. Three-dimensional virtual dissection at CT colonography: unraveling the colon to search for lesions. RadioGraphics 2006; 26:1669-1686

31. Pickhardt PJ. Differential diagnosis of polypoid lesions seen at CT colonography (virtual colonoscopy). RadioGraphics 2004; 24:1535-1559

32. Pickhardt PJ. Three-dimensional endoluminal CT colonography (virtual colonoscopy): comparison of three commercially available systems. AJR 2003; 181:1599-1606

33. Pickhardt PJ, Taylor AJ, Gopal DV. Surface visualization at 3D endoluminal CT colonography: degree of coverage and implications for polyp detec- 


\section{Pickhardt}

tion. Gastroenterology 2006; 130:1582-1587

34. Pickhardt PJ. Translucency rendering in 3D endoluminal CT colonography: a useful tool for increasing polyp specificity and decreasing interpretation time. AJR 2004; 183:429-436

35. Pickhardt PJ, Lee AD, McFarland EG, Taylor AJ. Linear polyp measurement at CT colonography: in vitro and in vivo comparison of two-dimensional and three-dimensional displays. Radiology 2005; 236:872-878

36. Pickhardt PJ, Lehman VT, Winter TC, Taylor AJ. Comparison of polyp volume versus linear size measurement at CT colonography: implications for noninvasive surveillance of unresected colorectal lesions. AJR 2006; 186:1605-1610

37. Pickhardt PJ, Choi JR, Nugent PA, Schindler WR. The effect of diagnostic confidence on the probability of optical colonoscopic confirmation for potential polyps detected at CT colonography: prospective assessment in 1339 asymptomatic adults. AJR 2004; 183:1661-1665

38. Zalis ME, Barish MA, Choi JR, et al. CT colonog- raphy reporting and data system: a consensus proposal. Radiology 2005; 236:3-9

39. Pickhardt PJ. The natural history of colorectal polyps and masses: rediscovered truths from the barium enema era. AJR 2007; 188: 619-621

40. Pickhardt PJ. CT colonography (virtual colonoscopy) for primary colorectal screening: challenges facing clinical implementation. Abdom Imaging 2005; 30:1-4

41. Pickhardt PJ, Nugent PA, Mysliwiec PA, Choi JR, Schindler WR. Location of adenomas missed at optical colonoscopy. Ann Intern Med 2004; 141:352-359

42. Bond JH. Clinical relevance of the small colorectal polyp. Endoscopy 2001; 33:454-457

43. Sanford MS, Pickhardt PJ. Diagnostic performance of primary 3-dimensional computed tomography colonography in the setting of colonic diverticular disease. Clin Gastroenterol Hepatol 2006; 4:1039-1047

44. Pickhardt PJ, Nugent PA, Choi JR, Schindler WR. Flat colorectal lesions in asymptomatic adults: im- plications for screening with CT virtual colonoscopy. AJR 2004; 183:1343-1347

45. Pickhardt PJ. High-magnification chromoscopic colonoscopy: caution needs to be exercised before changing screening policy. (reply) AJR 2006; 186:577-578

46. Pickhardt PJ, Choi JR, Hwang I, Schindler WR. Nonadenomatous polyps at CT colonography: prevalence, size distribution, and detection rates. Radiology 2004; 232:784-790

47. Prout TM, Taylor AJ, Pickhardt PJ. Inverted appendiceal stumps simulating large pedunculated polyps at screening CT colonography. AJR 2006; 186:535-538

48. Lee AD, Pickhardt PJ, Gopal DV, Taylor AJ. Venous malformations mimicking multiple mucosal polyps at screening CT colonography. AJR 2006; 186:1113-1115

49. Pickhardt PJ, Choi JR. Adenomatous polyp obscured by small-caliber rectal catheter at CT colonography: a rare diagnostic pitfall. AJR 2005; $184: 1581-1583$

\section{APPENDIX I: Potential Pitfalls in Interpretation of CT Colonographic Images}

Presence of residual feces, especially if untagged

Incomplete luminal distention

Presence of thickened or complex folds

Extrinsic impression

Presence of polyps coated with contrast material

Presence of the following conditions:

- Diverticular disease

- Invasive cancer (annular masses)

- Appendiceal lesions

- Submucosal lesions

- Anorectal lesions, such as internal hemorrhoids and hypertrophic anal papillae

- Flat and carpet lesions

- Nonadenomatous polyps

- Intraluminal foreign bodies, such as pills and capsules

- Pedunculated polyps (positional shift)

Recognition of the ileocecal valve

Presence of the rectal catheter

Polyp measurement

Presence of cleansing and subtraction artifacts

Spatial distortion on nonstandard 3D views 